\title{
A RADICAL ALGEBRA WITHOUT DERIVATIONS
}

\author{
DONALD J. NEWMAN
}

Wermer and Singer [1] have shown that in a semi-simple commutative Banach algebra there exist no nontrivial derivations, a derivation being a bounded linear operator $D$, taking the algebra into itself, with the additional property that

$$
D(u \cdot v)=u \cdot(D v)+(D u) \cdot v .
$$

Wermer has conjectured the following converse: If a commutative Banach algebra has no nontrivial derivations then it is semi-simple.

A weaker statement is: If a commutative Banach algebra is all radical [i.e. $x^{n} \rightarrow 0$ for all $x$ ] then it has a nontrivial derivation.

In this note we show that even this weaker statement is false.

We choose a fixed sequence $\lambda_{n}, n=1,2, \cdots$, of non-0 complex numbers, and consider the following algebraic system $\delta$.

The elements are those formal power series

$$
a(t)=\sum_{n=1}^{\infty} a_{n} t^{n} \text { for which } \sum\left|a_{n}\right|\left|\lambda_{n}\right|^{n}<\infty .
$$

Addition, multiplication, and multiplication by scalars is as usual.

Consider now the following properties
$\mathrm{A}:\left|\lambda_{n}\right| \geqq\left|\lambda_{n+1}\right|, n=1,2, \cdots$,
$\mathrm{B}: \lambda_{n} \rightarrow 0$,
C: $n^{\epsilon}\left|\lambda_{n+1}\right|^{n+1} /\left|\lambda_{n}\right|^{n} \rightarrow \infty$ for any fixed $\epsilon>0$.

We prove the following lemmas:

LeMmA 1. $\mathrm{A} \Rightarrow \mathrm{S}$ is a Banach algebra under the norm $\|a\|$ $=\sum\left|a_{n}\right|\left|\lambda_{n}\right|^{n}$.

LemmA 2. $\mathrm{A}$ and $\mathrm{B} \Rightarrow \mathrm{S}$ is all radical.

Lemma 3. A and $\mathrm{C} \Rightarrow S$ has no nontrivial derivations.

Proof 1. Since $S$, under this norm is isomorphic and isometric to $l^{1}$ under the correspondence

$$
\sum_{n=1}^{\infty} a_{n} t^{n} \leftrightarrow\left\{a_{n}\left(\lambda_{n}\right)^{n}\right\}
$$

$\mathcal{S}$ is clearly a Banach space. Also

Received by the editors November 5, 1958. 


$$
\begin{aligned}
\|a b\| & =\left\|\sum_{n=2}^{\infty}\left(\sum_{m+k=n} a_{m} b_{k}\right) t^{n}\right\| \\
& =\sum_{n=2}^{\infty}\left|\sum_{m+k=n} a_{m} b_{k}\right| \cdot\left|\lambda_{n}\right|^{n} \\
& \leqq \sum_{m, k}\left|a_{m}\right|\left|b_{k}\right|\left|\lambda_{m+k}\right|^{m+k}=\sum_{m, k}=\left|a_{m}\right|\left|b_{k}\right|\left|\lambda_{m+k}\right|^{m}\left|\lambda_{m+k}\right|^{k} \\
& \leqq \sum_{m, k}\left|a_{m}\right|\left|b_{k}\right|\left|\lambda_{m}\right|^{m} \cdot\left|\lambda_{k}\right|^{k} \text { (by A.) } \\
& =\|a\| \cdot\|b\|
\end{aligned}
$$

and so is a Banach algebra.

Proof 2. It is known that the radical $R$, is a closed subalgebra. But now $\left\|t^{n}\right\|{ }^{1 / n}=\left|\lambda_{n}\right|$ and by $\mathrm{B}$ this $\rightarrow 0 . \therefore t \in R$, by the algebraic closure of $R, P(t) \in R, P$ any polynomial, $\therefore$ by topological closure, all

$$
\begin{aligned}
a(t) \in R \cdot\left[\left\|a(t)-\left(a_{1 i}+\cdots a_{N} t^{N}\right)\right\|\right. & \\
& \left.=\sum_{n=N+1}^{\infty}\left|a_{n}\right|\left|\lambda_{n}\right|^{n} \rightarrow 0 \text { with } N\right] .
\end{aligned}
$$

Proof 3. Since, as in the above parenthetical remark, the polynomials are dense in $\delta$ it suffices to prove that $D(t)=0$ for $D$ any derivation, for it would then follow that $D(P(t))=P^{\prime}(t) D t=0$ and so $D(a(t))=0$ or $D$ is trivial.

Let

$$
D(t)=\sum_{m=1}^{\infty} c_{m} t^{m}
$$

Then

$$
D\left(t^{n}\right)=n t^{n-1} D(t)=n \sum_{k=0}^{\infty} c_{k+1} t^{k+n}
$$

$\therefore$ for any fixed $k=0,1, \cdots$

$$
\left\|D\left(t^{n}\right)\right\| \geqq n\left|c_{k+1}\right|\left|\lambda_{k+n}\right|^{k+n},
$$

on the other hand, $D$ being bounded,

$$
\begin{aligned}
\left\|D\left(t^{n}\right)\right\| & \leqq M\left\|t^{n}\right\|=M\left|\lambda_{n}\right|^{n}, \\
\therefore\left|c_{k+1}\right| & \leqq M\left|\lambda_{n}\right|^{n} / n\left|\lambda_{n+k}\right|^{n+k} .
\end{aligned}
$$

Now as $n \rightarrow \infty$ it follows from $\mathrm{C}$ that the right side $\rightarrow 0, \therefore c_{k+1}=0$, 
this holding for each $k=0,1, \cdots$ it follows that $D(t)=0$.

We now see that a counterexample to Wermer's conjecture is afforded us once we note that conditions A, B, C are not contradictory. This is clear, however, since e.g.

$$
\lambda_{n}=\frac{1}{\log (n+1)}
$$

satisfies all three of them.

\section{REFERENCE}

1. I. M. Singer and J. Wermer, Derivations on commutative normed algebras, Math. Ann. vol. 129 (1955) pp. 260-264.

Massachusetts Institute of Technology and

BROWN UNIVERSITY 